\title{
EL TRABAJO SOCIOEDUCATIVO EN PROYECTOS DE INCORPORACIÓN DE LAS TIC EN LOS CENTROS EDUCATIVOS: UNA EXPERIENCIA EN EL SISTEMA EDUCATIVO PÚBLICO DE EL SALVADOR \\ Luis González Minero CENICSH luis.minero@mined.gob.sv
}

Recepción:

12 de enero de 2018

Aceptación:

26 de enero de 2018 


\section{RESUMEN}

Este artículo sistematiza el trabajo socioeducativo impulsado desde el Viceministerio de Ciencia y Tecnología (Ministerio de Educación), el cual tiene como objetivo coadyuvar al proceso de incorporación de las TIC en las aulas de clases de las instituciones educativas públicas de El Salvador. En ese sentido, el análisis vertido en las siguientes páginas señala, por un lado, las experiencias que se han ganado y que han servido para retroalimentar y reconfigurar el trabajo (a lo largo de más o menos cinco años) en diferentes proyectos y programas que integran las TIC en educación: asambleas con padres y madres de familia, talleres de trabajo con líderes comunitarios, entrevistas a docentes, directores de centros educativos y líderes locales, diagnósticos socioeducativos de las comunidades educativas involucradas en Proyectos TIC y visitas de seguimiento al proceso de incorporación de las TIC dentro del proceso educativo.Y, por el otro, se establecen algunas conclusiones y se proyectan expectativas y estrategias sobre el camino que debería tomar este tema.

\section{Palabras Clave}

Trabajo socio-educativo, TIC, apropiación de la tecnología, comunidad educativa, globalización.

\section{ABSTRACT}

The present work systematizes socioeducational work promoted from the Vice Ministry of Science and Technology (Ministry of Education), which aims to contribute to the process of incorporating ICT into the classrooms of educational institutions public of El Salvador. In that sense, the analysis poured into the following pages points out, on the one hand, the experiences they have won and they have served to provide feedback and reconfigure work (throughout of about five years) in different projects and programs that integrate ICT in education: assemblies with parents and mothers of family, workshops with leaders community, interviews with teachers, directors of educational centers and local leaders, socio-educational diagnoses of the communities educational involved in Projects ICT and follow-up visits to the process incorporation of ICT within the process educational. And, on the other, some conclusions and expectations are projected and strategies on the path that should take this matter.

\section{Keywords}

Socio-educational work, ICT, technology appropriation, educational community, globalization. 


\section{EL TRABAJO SOCIOEDUCATIVO EN PROYECTOS DE INCORPORACIÓN DE LAS TIC EN LOS CENTROS EDUCATIVOS: UNA EXPERIENCIA EN EL SISTEMA EDUCATIVO PÚBLICO DE EL SALVADOR \\ Luis González Minero CENICSH luis.minero@mined.gob.sv}

\section{Introducción}

Este artículo posee la siguiente estructura: de manera sucinta se hace un breve planteamiento sobre la línea teórica del tema socioeducativo, la manera en que ha sido trabajado en El Salvador y un análisis del camino recorrido en cuanto a proyectos y programas de incorporación de las TIC, que buscan reducir la brecha digital en el sistema educativo público. Al final, se establecen algunas conclusiones sobre lo que se ha hecho y, de manera muy general, se hacen algunas proyecciones sobre el camino que se debe seguir. En alguna medida, lo que se ha buscado es marcar una escisión en lo que ha sido el trabajo socioeducativo; es decir, se hace una revisión y un balance del trabajo realizado, para después generar proyecciones que ayuden a fortalecer acciones programadas y retroalimentar nuevas estrategias de trabajo.

En primer lugar, es necesario establecer qué se entiende por trabajo socioeducativo. Para ello, se retoman algunas ideas consideradas prioritarias 
en torno a este tema y, de ahí, se puntualizan los principales elementos abordados y contextualizados hasta este momento. Luego, se articula el trabajo socioeducativo con la incorporación de las TIC en los centros educativos públicos; en otras palabras, sobre la manera en que se fortalecen los procesos de empoderamiento, sensibilización educativa y apropiación de la tecnología dentro de las comunidades educativas. Para finalizar, se parte de una reflexión y análisis de las acciones ejecutadas y las experiencias acumuladas para establecer algunas conclusiones sobre lo que ha sido el trabajo socioeducativo en proyectos de incorporación de TIC en los centros educativos, y la relevancia de este tema para el éxito de este tipo de proyectos que se ejecutan dentro del Ministerio de Educación (MINED). Pero también, de una manera preliminar, para plantear adecuaciones a las estrategias y acciones que deben continuar ejecutándose.

Las experiencias que se retoman en este artículo parten de cuatro momentos de trabajo: el primer momento es la articulación del trabajo entre la escuela y la comunidad (organización de la comunidad educativa); el segundo momento es el análisis de los contextos socioeducativos; el tercero constituye la elaboración de contenidos socioeducativos para capacitación de docentes en TIC; y, el último, consiste en el proceso de sistematización de acciones para la apropiación de las TIC dentro de los centros educativos (seguimiento y asistencia socioeducativa). Una etapa final de los cuatro momentos corresponde a una oportunidad para revisar, retroalimentar y redefinir acciones en el trabajo socieducativo que se implementa, buscando, así, cimentar una base que permita el empoderamiento, la sensibilizacion educativa y una adecuada apropiación de la tecnología dentro de la comunidad educativa en distintos contextos.

\section{El trabajo socioeducativo}

La didáctica a menudo se ha considerado una disciplina demasiado identificada con la escuela. Esto plantea una visión restrictiva de la misma y no reconoce la riqueza educativa que se puede obtener más allá de las paredes 
del aula y, sobre todo, de la importancia del conocimiento didáctico para la educación social. ${ }^{1}$ Desde la perspectiva que se quiere señalar en este trabajo, lo anterior cobra sentido con la realidad del mundo en que vivimos, ya que se parte de que toda educación es social y, por lo tanto, constituye un proceso que interrelaciona los ámbitos público y privado del ser humano, de lo social y lo individual en la acción humana. Es decir, sobre el cuido de la persona misma, pero también de aquello que le rodea. ${ }^{2}$

Para Ortega, «la educación supone una progresiva y continua configuración de la persona para ser y convivir con los demás, que se desarrolla a lo largo de la vida y no se limita a la escuela». ${ }^{3}$ Bajo esta idea de educación (a lo largo de la vida) es donde se inserta la educación social y esta podría entenderse, por un lado, como la promoción y dinamización de las condiciones educativas presentes en la vida social y, por otro, sobre la posición que ocupa la persona para hacer frente a la compleja dinámica social y sus conflictos con responsabilidad ética. ${ }^{4}$

\footnotetext{
${ }^{1}$ Nuria G. Freixes y Artur Parcerisa-Aran, «La intervención socioeducativa desde una mirada didáctica», Revista Edetania No 45 (2014): 57, disponible en: https://dialnet.unirioja.es/servlet/articulo?codigo $=5010267$

${ }^{2}$ Para ampliar esta idea se pueden revisar los trabajos sobre la ética del cuidado de sí, de M. Foucault, y la idea de cuidado, de L. Boff. Veáse Michel Foucault, «La ética del cuidado de sí como práctica de la libertad», diálogos con H. Becker, R. Fornet-Betancourt, A. GómezMüller, 20 de enero de 1984, en Estética, Ética y Hermeneutica, Obras esenciales volumen III (Buenos Aires: PAIDOS, 1999), 393-416. Y también, Macarena Álamo Santos, «La idea de cuidado en Leonardo Boff», Revista Tales, No 4 (2011): 243-253, disponible en: https:// revistatales.files.wordpress.com/2012/05/243_nro4nro-4.pdf

3 José Ortega, «Pedagogía Social, realidades actuales y perspectivas de futuro», Revista de Educación $\mathrm{N}^{\circ} 336$ (2005): 114, disponible en: http://www.revistaeducacion.mec.es/re336/ re336_07.pdf

${ }^{4}$ Es posible entender la educación social como una acción promotora y dinamizadora de una sociedad que eduque y una educación que integre a los individuos y, a la vez, que ayude al diálogo y al encuentro de soluciones pacíficas frente a los conflictos sociales. Por otro lado, las condiciones educativas deben entenderse como los fenómenos, procesos y vínculos que se establecen dentro de un determinado espacio educativo (sea formal o no), y que tienen como objetivo la transmisión y construcción de conocimientos, vivencias, ideas y valores que forjan identidades y definen sistemas sociales y culturales. En ese sentido, es necesario profundizar sobre el sentido que toman las condiciones educativas, es decir, sobre su producción y reproducción en determinados contextos.
} 
Partiendo de lo anterior, se busca que la persona —además de adquirir conocimientos - sea capaz de utilizar ese conocimiento, articulándolo con sus experiencias subjetivas y objetivas de vida social e individual, para poderse posicionar y gestionar alternativas que brinden soluciones a los problemas que le afectan. Es decir, desde este punto, la educación no puede limitarse a una experiencia inmersa en cuatro paredes, sino que obedece a un proceso interactivo y dialéctico entre vida personal y social que se vierte en acciones enfocadas a mejorar la calidad de vida individual y de todas las personas.

Cuando a la educación se le aparta de su condición social y se le restringe a una dimensión únicamente individual (en la que no se logra hacer el vínculo entre conocimiento y práctica) se tiene una educación incompleta, y es ahí donde radica la importancia de la intervención socioeducativa. Para Freixes y Parcerisa-Aran, esta brinda una «[...] intencionalidad educativa [...] orientada a favorecer la autonomía de la persona en el planteamiento y en la resolución de sus problemáticas». ${ }^{5}$

Se debe hacer notar que el abordaje socioeducativo es bastante amplio y complejo. Por lo tanto, es prudente concretarlo a situaciones específicas, tal es el caso del proceso de incorporación de las TIC dentro del contexto educativo. Se parte de que dicho proceso resulta algo nuevo para la mayoría de los contextos educativos y, por tanto, requiere del establecimiento de acuerdos y corresponsabilidades entre los diferentes actores participantes que se extiendan más allá de las paredes del aula y la escuela, de manera que la apropiación de la tecnología sea exitosa y obtenga buenos resultados.

Desde esta perspectiva, el trabajo socioeducativo se orienta a mejorar las condiciones de trabajo y las relaciones entre los actores que participan dentro del proceso educativo donde se incorporan las TIC. De tal manera que se establezcan mecanismos de apropiación de la tecnología coherentes con la realidad de cada contexto educativo, y que además cuenten con el consenso de los miembros de la comunidad educativa. En definitiva, el

\footnotetext{
${ }^{5}$ Nuria G. Freixes y Artur Parcerisa-Aran, «La intervención socioeducativa desde una mirada didáctica», 57.
} 
trabajo socioeducativo permite dar un giro al tema de las tecnologías, pues pone el énfasis en las personas que utilizan el recurso tecnológico y en la manera en que lo hacen, y no en el recurso en sí mismo. En ese sentido, es necesario construir las condiciones que permitan a las personas apropiarse de la tecnología de una manera adecuada, esto ocurre bajo dos principios básicos: uso responsable y cuido del recurso tecnológico. Es bajo estos principios que el trabajo socioeducativo cobra relevancia.

\section{Articulación socioeducativa y TIC}

Actualmente las sociedades se encuentran impactadas fuertemente por los avances tecnológicos; ello, en mayor o menor medida, contribuye a situarlas en un estado de constante cambio. Algunos autores reconocen este fenómeno como «shock del futuro", lo cual tiene que ver con las «[...] dificultades que se suscitan en las sociedades en cuanto a la forma en que procesan, comprenden e incluso sobreviven al incesante estado de cambio y los problemas que devienen con éstos». ${ }^{6}$

Este mundo contemporáneo y globalizado se encuentra expuesto a un proceso de extrema individualización; en él, los seres humanos tienden a replegarse y enfrascarse dentro de un pequeño mundo y a buscar salvación confiando solamente en sus recursos individuales. Estos sucesos no pasan desapercibidos, sino que marcan su influencia en diferentes ámbitos de la vida; por ejemplo, las TIC inmersas en ese contexto llevó a Bauman a proponer que «las redes sociales son una trampa», pues estas tienden a alejar a las personas de la realidad y sumergirlas en una especie de ficción dónde son capaces de controlar con quienes se relacionan — una persona fácilmente puede añadir y eliminar amigos-, por lo tanto, las redes sociales crean un sustituto en el cual las habilidades sociales no son necesarias para abordar la complejidad de las relaciones humanas:

\footnotetext{
${ }^{6}$ Organización de las Naciones Unidas para la Educación, la Ciencia y la Cultura (UNESCO), Las Tecnologías de la información y la comunicación en la enseñanza. Manual para docentes o cómo crear nuevos entornos de aprendizaje abierto por medio de las TIC (Francia: División de Educación Superior UNESCO, 2005), 15.
} 
Las redes sociales no enseñan a dialogar porque es tan fácil evitar la controversia... Mucha gente usa las redes sociales no para unir, no para ampliar sus horizontes, sino al contrario, para encerrarse en lo que llamo zonas de confort, donde el único sonido que oyen es el eco de su voz, donde lo único que ven son los reflejos de su propia cara. Las redes son muy útiles, dan servicios muy placenteros, pero son una trampa. ${ }^{7}$

Como se dijo anteriormente, las TIC avanzan a paso vertiginoso y el impacto que tienen en la vida humana es desmesurado. Por lo tanto, es necesario poner atención a la interacción y a la relación de las personas con ellas; es decir, en la manera en que se apropian de la tecnología. Este punto es la articulación entre las TIC y el trabajo socieducativo, ya que es este último el que otorga direccionalidad al uso que las personas dan a las TIC y, de esa manera, el sentido que se configura en el tipo de apropiación de la tecnología es lo que determina su utilización.

No es posible pensar únicamente en una especie de determinismo tecnológico. Diéguez entiende este como la capacidad de la tecnología de incidir de manera directa en el desarrollo social y económico de un determinado contexto y sobre el cual existe una ausencia de control de la tecnología por parte de la persona, ${ }^{8}$ sino se debe pensar en la idea de que las TIC se sumergen dentro de una dimensión humana y dentro de un proceso dinámico que se manifiesta al brindar oportunidades de diálogo, interacción y sinergia entre las personas. Dicho de otro modo, el interés se centra en la persona como actor que determina los usos y los sentidos que da a los objetos con los que interactúa y manipula dentro de un determinado contexto.

\footnotetext{
${ }^{7}$ Ricardo De Querol, «Zygmunt Bauman: Las redes sociales son una trampa», El País, enero 09, 2016, disponible en: https://elpais.com/cultura/2015/12/30/babelia/1451504427_675885. html

${ }^{8}$ Alejandro Diéguez, «El determinismo tecnológico: indicaciones para su interpretación», Argumentos de Razón Técnica No. 8. (Universidad de Málaga. España, 2005), disponible en https://sociotecno7.files.wordpress.com/2009/09/determinismo-tecnologico.pdf
} 


\section{Apropiación social de la tecnología: el trabajo socioeducativo articulado a las TIC en El Salvador}

La articulación de las TIC y el trabajo socioeducativo se da en un punto denominado apropiación social de la tecnología. La tecnología no puede desvincularse del contexto, ya que depende de la visión de mundo de los individuos que trabajan con ella y que le dan sentido y significado. Con ello, se propone que la tecnología debe ser adaptada para dar soluciones contextualizadas y acordes a las necesidades de la sociedad, y como ejercicio democrático en la construcción del conocimiento. Esta visión implica reconocer la existencia de múltiples saberes y la necesidad de construir espacios de inclusión y participación activa a los distintos actores sociales. ${ }^{9}$

Esta noción actúa de manera muy similar a la idea de llevar a cabo una especie de empoderamiento tecnológico, entendido como un proceso por medio del cual las personas utilizan la tecnología como una herramienta que les permite fortalecer sus capacidades, confianza, visión y protagonismo, desde la integración y la cohesión social para impulsar cambios y transformaciones en los hábitos, y que les lleva a asumir desde sí mismas la responsabilidad sobre su contexto y que encuentren en su uso un medio que apoye a mejorar su calidad de vida. El empoderamiento y la sensibilidad educativa forman elementos claves y fundamentales que otorgan sentido a la apropiación de la tecnología, ya que permiten a las personas la utilización de herramientas tecnológicas, no como una obligación o imposición, sino más bien de manera responsable tanto consigo mismas, como dentro de un determinado entorno social y cultural.

Para Dussel, la presencia de las nuevas tecnologías en las aulas ya no tiene vuelta atrás. Si hasta hace unos años podía pensarse que los medios digitales debían restringirse a algunas horas por semana o a algunos campos

\footnotetext{
${ }^{9}$ Claudia Aguirre Minvielle, «Definición de prácticas de Apropiación Social de la Ciencia y de la Tecnología, historia de una conversación", Revista Ciencia, tecnología y democracia: Reflexiones en torno a la apropiación social del conocimiento, Memorias del Foro-Taller de Apropiación Social de la Ciencia, la Tecnología y la Innovación, ed. Pérez Bustos, Tania; Lozano Borda, Marcela (Universidad EAFIT. Colciencias. Medellín, Colombia, 2011), 181. pp. 177-184.
} 
de conocimiento, hoy es dificil (si no imposible) poner límites a su participación en los procesos de enseñanza y aprendizaje. ${ }^{10}$ En ese sentido, la apropiación social de la tecnología dentro del contexto educativo salvadoreño busca cambios en dos direcciones: la primera, cambios en los hábitos dentro de los procesos educativos; $\mathrm{y}$, segundo, cambios en las formas de organización y las relaciones entre los miembros de la comunidad educativa.

Cuando se habla de cambios en los hábitos de los procesos educativos, se tiene que tomar en cuenta aquello relacionado a las nuevas prácticas que sugiere la incorporación de las TIC dentro de las relaciones que se viven en el contexto educativo. Es decir, garantizar el uso adecuado y responsable de los recursos tecnológicos, con fines educativos y con miras a fortalecer las capacidades y los conocimientos de docentes y estudiantes. Por el otro lado, sobre los cambios en la organización y las relaciones entre los miembros de la comunidad educativa, se necesita generar una estructura social sólida que brinde seguridad a los recursos tecnológicos y que además sistematice acciones y estrategias donde se acuerdan corresponsabilidades entre los diferentes actores que participan dentro del proceso educativo.

Como todo proceso, la apropiación social de la tecnología persigue un fin. En definitiva, para el caso salvadoreño, dicho fin se centra en la construcción de un proyecto educativo que incorpore las TIC dentro del proceso educativo. Metodológicamente, la apropiación social de la tecnología sistematiza experiencias, a partir de la participación activa y protagónica de quienes forman parte de dicha experiencia. Por esta razón es necesario que las personas asuman responsabilidades y compromisos en los que se definan acciones y estrategias claras para la construcción del proyecto que permita la adecuada apropiación social de la tecnología; es decir, en esta parte se somete a un diálogo sobre las corresponsabilidades y el papel de los participantes (director, docentes, estudiantes, padres y madres de familia, entre otros), tanto a nivel individual como social. Lo anterior también permite la búsqueda de apoyos — municipalidad, empresa privada, ONG, entre otros - y el lideraz-

\footnotetext{
${ }^{10}$ Inés Dussel, «Aprender y enseñar en la cultura digital», VII Foro Latinoamericano de Educación; educación y nuevas tecnologías: los desafíos pedagógicos ante el mundo digital, ed. Dussel, Inés y Quevedo, Luis Alberto (Buenos Aires: Santillana, 2011).
} 
go y organización de los actores que permitan el ejercicio ciudadano y la apropiación de su propio desarrollo educativo, considerando los usos adecuados y cuido de los recursos tecnológicos.

Los cambios señalados anteriormente hay que verlos con mucha atención, pues los problemas que atañen a la educación del país son complejos y no dependen únicamente de las TIC para solucionarlos: requieren que se tomen en cuenta también la cultura, los significados y los aspectos simbólicos que las personas construyen en determinado contexto y sobre los objetos con los que interactúan en dicho contexto. Dussel señala que:

[...] las nuevas tecnologías tienen lógicas y modos de configurar el conocimiento muy diferentes a los de la escuela. Las primeras funcionan en base a la personalización, la seducción y el involucramiento personal y emocional, y suelen ser muy veloces y con una interacción inmediata. La escuela, en cambio, es una institución basada en el conocimiento disciplinar, más estructurada, menos exploratoria, y con tiempos y espacios determinados de antemano, más lentos y menos porosos. Cabe esperar entonces un proceso de negociación y de reacomodamiento de la institución escolar que no será automático ni inmediato, y que no debería ser leído solo como resistencia al cambio. ${ }^{11}$

Para Andrade y Campo-Redondo, el reto que tiene la apropiación social de la tecnología consiste en llevar a cabo una articulación donde lo social y lo tecnológico puedan encontrarse para construir una relación explícita entre ellas; ${ }^{12}$ en otras palabras, una adecuada apropiación de la tecnología dentro del sistema educativo pasa por ubicar a docentes y estudiantes como elementos indispensables que utilizarán las herramientas TIC para mejorar el proceso educativo, y que además son agentes sujetos a constantes históricas y culturales; y si, bien es cierto, pueden existir momentos de resistencia,

\footnotetext{
${ }^{11}$ Inés Dussel, «Aprender y enseña en la cultura digital», 13.

${ }^{12}$ Jesús Alberto Andrade y María Campo-Redondo, «Apropiación social de las tecnologías de información: políticas públicas para la participación ciudadana», Quórum Académico, Volumen 9, No 1 enero-julio 2012 (Venezuela: Universidad del Zulia, 2012), 52-68.
} 
ello se debe entender como un proceso de tránsito en diferentes niveles de apropiación que conlleva a la reconfiguración de símbolos y significados sobre la tecnología.

\section{Experiencia del trabajo socioeducativo y las TIC en El Salvador}

En el país, las primeras experiencias en este tema se dan con la implementación del Programa Cerrando la Brecha del Conocimiento (CBC), impulsado por el Viceministerio de Ciencia y Tecnología, a partir de 2009. Con este se buscaba elevar la calidad de la educación por medio de la ampliación del acceso a las TIC para que sean integradas como herramienta pedagógica en el salón de clases. Es decir que, directa o indirectamente, se apoyara al desarrollo de competencias cognitivas y tecnológicas para que los beneficiarios puedan — en la medida de su propio desarrollo- contribuir al progreso socioeducativo y cultural de sus comunidades. ${ }^{13}$ Cabe señalar que este programa se desarrollaba, principalmente, en centros educativos con rezago académico y ubicados en municipios de extrema pobreza.

A partir de 2014, el trabajo socioeducativo fue incluido dentro de las acciones que se implementan con el Programa «Una Niña, Un Niño, Una Computadora». De esta manera, el tema socioeducativo ya no se trabaja con grupos particulares de escuelas, sino que amplía su cobertura y se dirige a todos aquellos centros educativos con acceso a recursos tecnológicos. Al llegar a este punto, el interés del trabajo socioeducativo se centra en el acercamiento de las escuelas con la familia y la comunidad; de manera que la comunidad educativa participe activamente y responsablemente en la definición, dinamización y ejecución de los planes de desarrollo educativos, principalmente de aquellos referentes al uso responsable y cuido de los recursos tecnológicos.

Desde su inicio, el trabajo socioeducativo ha mutado y se ha reconfigurado a partir tanto de las experiencias generadas, como de los rumbos

\footnotetext{
${ }^{13}$ Ministerio de Educación, «Plan de Acción del Programa Cerrando la Brecha del Conocimiento». (El Salvador: MINED, 2009).
} 
que ha tomado la entrega de recursos tecnológicos a los centros educativos. No obstante, las diferentes acciones que se han ejecutado a lo largo de estos años se pueden organizar en cuatro momentos de trabajo: organización de la comunidad educativa, análisis de los contextos socioeducativos, diseño de contenidos socioeducativos para capacitación de docentes en TIC y seguimiento y asistencia socioeducativa. Al finalizar el desarrollo de los diferentes momentos, se abre una oportunidad para llevar a cabo un proceso de revisión, retroalimentación y redefinición de acciones.

Es importante señalar que estos momentos se interrelacionan, y cada uno de ellos corresponde a un nivel o profundidad de acercamiento en el trabajo socioeducativo y los actores presentes en cada contexto educativo, para alcanzar diferentes niveles de apropiación de la tecnología. De esta manera, no es posible ver cada momento de manera aislada, sino que, en definitiva, configuran una unidad sistematizada de interdependencias que buscan dar sentido al nivel de apropiación que se constituye en torno a las TIC dentro de diferentes contextos educativos.

- Organización de la comunidad educativa: aquí se busca una articulación del trabajo entre la escuela y la comunidad, pero también, entre redes de centros educativos. Para ello se realizan visitas de campo con el fin de conocer e identificar diferentes actores claves que potencian el desarrollo educativo y, específicamente, la apropiación de la tecnología dentro de los centros educativos. A través de las diferentes visitas, es posible observar y percibir una parte de la realidad social y los tipos de relaciones sociales entre los diferentes actores que integran las diferentes comunidades educativas. También se llevaron a cabo diferentes asambleas generales en los centros educativos, con el propósito de fortalecer los lazos entre la comunidad y la escuela, entablar un diálogo y generar acuerdos que coadyuven a mejorar las condiciones educativas de las comunidades; específicamente, en torno al uso y cuido de los recursos tecnológicos disponibles en los centros educativos. Otro aspecto importante 
es que se realizan talleres de trabajo en los centros educativos, los cuales son dirigidos a personas involucradas directamente con el desarrollo educativo y, así, obtener información y valoraciones sobre la comunidad y el quehacer educativo de los diferentes contextos, motivar y sensibilizar sobre las necesidades de definir estrategias de trabajo enfocadas al uso responsable y cuido del recurso tecnológico.

- Análisis de los contextos socioeducativos: se trata de un análisis para sondear el contexto social y cultural en el que se encuentran las comunidades y los centros educativos. Así, se parte del hecho de que la institución educativa no está aislada, sino que forma parte de la comunidad y de los elementos culturales presentes en ella; estos elementos impactan en la manera en que las personas se apropian de la tecnología. En este momento se interactúa con diferentes actores (director, docente, estudiante, padre y madre, liderazgos comunitarios, entre otros), tanto fuera como dentro del espacio educativo, y se obtiene información referente a significados, simbolismos y representaciones de cada uno de ellos frente a las TIC; después la información se articula y se reconstruye a nivel teórico el contexto. En este momento, se encuentran puntos de convergencia y divergencia en el trabajo que realizan los diferentes centros educativos.

- Diseño de contenidos socioeducativos para capacitación docente en TIC: la base para el diseño de estos contenidos parte de tres etapas: conceptualización, diseño e implementación. En la primera etapa, se dan a conocer nociones teóricas sobre la apropiación social de la tecnología; además, se motiva a la reflexión sobre la necesidad de la actualización docente dentro de un mundo donde las TIC juegan un papel importante en el manejo y construcción del conocimiento. La segunda trata sobre el diseño de un proceso que contenga y sistematice estrategias y líneas de acción que deberán regir el desarrollo de la apropiación de la tecnología en las instituciones educativas. La 
última parte de la formación consiste en la implementación, seguimiento y evaluación de acciones orientadas a la apropiación de la tecnología en el contexto educativo. Tales acciones deben apuntar al mejoramiento de los procedimientos institucionales orientados hacia una adecuada integración de las TIC en el contexto educativo; es decir, al uso educativo y el cuido de los recursos tecnológicos disponibles.

- Seguimiento y asistencia socioeducativa: este momento constituye un proceso de revisión, recopilación y sistematización de acciones para la apropiación de las TIC implementadas dentro de los centros educativos. Se visitan centros educativos con el propósito de conocer el funcionamiento que tienen los mecanismos institucionales de trabajo que garantizan el adecuado uso y el cuido del recurso tecnológico entre los miembros de la comunidad educativa. En las visitas se detectan buenas prácticas que pueden ser retomadas para implementar en otros contextos o, por el contrario, si hay hallazgos negativos se redefinen o redireccionan acciones que permitan la apropiación de tecnología. Aquí, también es posible generar y potenciar redes de trabajo entre centros educativos (a partir de la identificación de convergencias o divergencias), de tal manera que pueda existir transferencias de conocimientos y experiencias.

En cada uno de los momentos se busca — en alguna medida - reconstruir la realidad socioeducativa de las comunidades, desde la perspectiva y con la participación de los diferentes actores que intervienen a nivel local. En definitiva, se busca crear, desde y con las comunidades educativas, las condiciones mínimas necesarias para la incorporación, el uso adecuado y el cuido de los recursos tecnológicos; poniendo énfasis en que las estrategias educativas generadas dentro de cada contexto deben partir de la identidad, saberes y motivaciones propios de cada persona y cada grupo. 


\section{Conclusiones del trabajo socioeducativo en proyectos TIC}

Los elementos que conforman la sociedad se mueven en diferentes esferas (social, educativa, cultural, económica y política) que no pueden ser independientes. Los actores sociales tienen el papel de dinamizar procesos y son ellos quienes tienen la capacidad de transformar las estructuras en las diferentes esferas; por tanto, el trabajo socioeducativo enfatiza brindar autonomía e independencia en las acciones. En ese sentido, el tema socioeducativo y las TIC debe estar enfocado en la incorporación y el involucramiento de los diferentes actores, a partir de los intereses a nivel local y — de esa manera- motivar y dinamizar procesos que amplíen el acceso a la información y que brinden herramientas cognitivas que permitan mejorar las condiciones sociales y educativas de los habitantes. Hay un interés explícito por lograr que sean las personas las gestoras de su propio desarrollo y porque ellas tracen la ruta que se debe seguir para alcanzarlo.

A partir de lo anterior, es posible establecer la importancia que cumple el análisis de la realidad social y cultural de las comunidades y los centros educativos como parte del trabajo socioeducativo. Este tema resulta clave para entender la dinámica e identificar las características de las relaciones al interior de los diferentes grupos, de las condiciones sociales que les rodean y de los significados y simbolismos que ellos configuran en torno a determinados elementos; y de ahí, potenciar la transferencia de conocimientos y experiencias por medio de redes de trabajo. Por consiguiente, al conocer el comportamiento real, los usos y significaciones sobre la tecnología (pero a su vez entendiéndolo como producto de la interacción de los factores objetivos y subjetivos en entornos diferenciados), es posible crear estrategias de trabajo reales acordes a cada contexto. Dichas estrategias se encaminan a motivar, incentivar y propiciar la participación y la corresponsabilidad de los diferentes actores a nivel local en torno a la tecnología.

Con el conocimiento de las relaciones que son características de cada lugar, es posible elaborar estrategias de abordaje a las comunidades y entre comunidades. Este abordaje debe ser enfocado de acuerdo a las necesidades que enfrenta cada centro escolar y al tipo de relaciones que se gestan 
al interior de los grupos en determinados contextos. Dentro de las relaciones se identifican los actores, sus prácticas y formas de percibir su entorno, además se canaliza el trabajo para construir procesos de fortalecimiento de los lazos sociales que unen a las personas y los grupos para llevar a cabo transformaciones sociales. De esta forma, el punto desde donde se lanzan las estrategias de coordinación - entre los diferentes actores sociales a nivel local - se enfoca en el uso y cuido de los recursos tecnológicos, y de ahí se derivan hacia otras direcciones como mejoramiento de infraestructura, gestión de procesos de capacitación, proyectos de colaboración entre escuela y comunidad, entre otros.

El tema socioeducativo, visto dentro del campo de las tecnologías educativas, conlleva un proceso de apropiación, el cual tiene que ver con el empoderamiento y la sensibilización educativa de los actores que integran la comunidad educativa; en consecuencia, si no se acepta la importancia y no se asume la responsabilidad y el compromiso hacia el uso adecuado y el cuido de los recursos tecnológicos, es casi imposible obtener un impacto positivo. Las personas deben ser conscientes sobre la importancia que tendría el aprendizaje y uso de tecnología en el mejoramiento de sus condiciones educativas; de lo contrario, es algo que no tendrá mayor relevancia y no trascenderá hacia los niveles de desarrollo social y educativo esperados, aunque es claro que esto debe ir acompañado de otros factores asociados a la ampliación del acceso a las TIC (como el caso del internet $\mathrm{u}$ otras herramientas) y el fortalecimiento de capacidades en el manejo de las mismas. De ahí la importancia de la apropiación social de la tecnología para gestionar transformaciones dentro de las comunidades.

En definitiva, el trabajo socioeducativo busca la articulación de las relaciones que se dan al interior de las esferas sociales y educativas en un determinado contexto, pero también de su interacción con la cultura, lo económico y lo político. El éxito de esta articulación y de sus relaciones es lo que permite o impide el crecimiento de dichas esferas, condicionando así el desarrollo, tanto a nivel individual como colectivo de las personas. Por lo tanto, una de las características de las estrategias que se construyen dentro del trabajo socioeducativo es que debe ser endógena, donde se tome en cuenta los sistemas de vida, intereses, expectativas, condiciones culturales y proyec- 
ciones que son característicos en cada grupo. Este hecho dará un sentido de pertenencia a las acciones y, en gran medida, garantiza una adecuada apropiación de la tecnología, hablando en términos educativos.

Cuando se habla de proyectos educativos que incorporan las TIC, el trabajo socioeducativo debe articularse con otras áreas de trabajo (prevención de violencia, desarrollo local, emprendedurismos, clubes de tareas o de otra índole, entre otros) y así generar nodos para la acción. Sería demasiado ambicioso e irracional pensar que el trabajo socioeducativo se desarrolla de manera aislada; si no, más bien, se encuentra en un continuo de influencia recíproca con diversas áreas con las que tiene convergencia, es ahí cuando surge la riqueza de este trabajo y la capacidad de potenciar dichos proyectos. El área socioeducativa funciona como una especie de red que contribuye a enlazar y consolidar puntos que conllevan a una adecuada apropiación social de la tecnología por parte de los grupos humanos. Es importante señalar que así como se puede tejer una red de trabajo a nivel de cada centro educativo - para el aprovechamiento de los recursos, tanto a nivel escolar como a los niveles personal, familiar y comunitario- también lo es conformar redes de centros educativos cercanos que serán estratégicas para empoderar y potenciar la organización de las comunidades educativas en el uso responsable y cuido que se debe dar a los recursos tecnológicos con que cuentan los centros educativos, y para compartir experiencias y conocimientos que dinamicen y fortalezcan las estrategias de trabajo.

La simbiosis entre centro educativo y comunidad y la articulación del trabajo en redes de centros educativos son necesarias para garantizar la sostenibilidad; pero ésta solo es posible garantizando una adecuada apropiación social de la tecnología, misma que (como ya se dijo antes) involucra procesos de empoderamiento tecnológico y de sensibilización educativa de los habitantes de las comunidades. Por un lado, el empoderamiento tecnológico consiste en un proceso por medio del cual las personas cambian y transforman sus hábitos, que les lleva a asumir desde sí mismas la responsabilidad sobre sus intereses y que encuentran en el uso de herramientas tecnológicas un medio de apoyo para mejorar las diferentes prácticas educativas en las que se ven inmersas. Por otro lado, la sensibilización educativa consiste en 
reflexionar sobre la importancia de la educación en las vidas de las personas, sobre su contexto y su historia, y sobre la incidencia y aplicabilidad de la tecnología para el desarrollo educativo. Estos procesos requieren ir dentro de un marco de cooperación, participación y corresponsabilidad activa de todos los actores sociales para solucionar las necesidades que enfrentan los centros educativos y mejorar las condiciones educativas.

De manera general, lograr una adecuada apropiación social de la tecnología fortalece los niveles organizativos al interior de las comunidades para mejorar sus condiciones educativas; además, fomenta la participación para la toma de decisiones y propicia la construcción de pensamiento crítico, creativo y propositivo para solucionar problemas que les afectan. Sin embargo, cabe señalar que el trabajo desempeñado hasta la fecha presenta algunos puntos débiles que impiden llevar a cabo plenamente todas las acciones descritas anteriormente. Entre estos:

- Alcance del trabajo socioeducativo y limitados recursos: en el país existen alrededor de 5,145 centros educativos públicos; alcanzar a todos requiere que se dispongan y optimicen recursos. Por lo tanto, es necesario que se construyan estrategias de trabajo donde los recursos disponibles sean optimizados y se potencie la capacidad institucional, principalmente de organizaciones, redes o alianzas. Hasta la fecha, una de las mayores limitantes ha sido establecer alianzas tanto a nivel interno de MINED (con otras unidades) como dentro de las comunidades. A nivel interno, el MINED tiene su propia dinámica, por lo general cada unidad se enfrasca dentro de su quehacer y en muy pocas ocasiones se logran coordinar acciones; en las comunidades sucede algo muy similar, en este caso escuela y comunidad son independientes y cuando se habla de comunidad se hace referencia al nivel organizativo que existe fuera de la escuela. La articulación se ha logrado de mejor manera en las comunidades, aunque con un alcance bastante limitado debido a que se tienen pocos recursos para cubrir los centros educativos del país. 
- Inseguridad en las comunidades: en los últimos años, el país se ha visto aquejado considerablemente por la inseguridad; esta situación presenta un riesgo latente tanto para las personas que viven y aquellas que visitan las comunidades, como para los recursos tecnológicos que se entregan a los centros educativos. La sensibilización pretende generar algún grado de conciencia sobre los beneficios educativos que se pueden obtener con los recursos disponibles, pero también de los vacíos que habrá si el equipo no se cuida.

- Desconfianza y apatía de las personas: esta limitante presenta un alto matiz político, sobre todo en la manera que ha sido manejado el Programa «Una Niña, Un Niño, Una Computadora» desde el Gobierno. Los discursos en muchos casos no han sido los mismos, ni tampoco la atención a las comunidades (este hecho está fuertemente vinculado con la falta de recursos y a las características que supone cada proyecto o entidad). Este hecho ha impactado fuertemente la percepción de la población sobre el programa y sus acciones, las cuales en algunos casos llegan a ser hasta rechazadas.

- Temor y falta de familiaridad con la tecnología: esta limitante se da particularmente en las personas de mayor edad. En este grupo de personas es posible observar un temor explícito hacia la utilización de herramientas tecnológicas, el cual tiene que ver con la falta de familiaridad con dichas herramientas (hay docentes que nunca han utilizado este tipo de herramientas o presentan muchas dificultades en su manejo). Otro aspecto importante de señalar es que la incorporación de la tecnología en los procesos educativos supone un cambio en la manera de hacer las cosas, sobre todo en la manera en que los y las docentes han percibido hasta este momento la educación —una educación centrada en la transmisión de conocimiento de docente a estudiante-, y por tanto, dotar de un recurso que fomente la participación, el diálogo, horizontalice las relaciones y no trans- 
mita (sino también construya) conocimiento, genera un sismo en los y las docentes.

Estos elementos que se han mencionado han dado como resultado que los recursos tecnológicos disponibles en los centros educativos sean desaprovechados, poco utilizados o dispuestos para otras tareas que no son precisamente educativas; asimismo, su conjugación resulta en una baja participación de los actores o un rechazo a las acciones que se implementan.

Si bien es cierto que la experiencia obtenida en la participación en diferentes proyectos y programas ha permitido ir puliendo esta herramienta de trabajo, es necesario tomar en cuenta el carácter dinámico de la sociedad, así como también añadir elementos que fortalecen o dan nuevo sentido a dicha herramienta; por tanto, debe ser un proceso en permanente revisión y actualización. Hasta este momento, los recursos tecnológicos vienen a funcionar como un punto de enlace desde donde se lanzan diferentes acciones para alcanzar una adecuada apropiación de la tecnología dentro de las comunidades. La tecnología por sí misma no es suficiente, requiere de capacidades sociales y culturales que echen a funcionar diferentes mecanismos y estrategias de desarrollo educativo, las cuales necesitan tiempo y esfuerzo. El camino es todavía largo, pero se ha logrado trazar una ruta y se deben encauzar las diferentes acciones en ella para obtener buenos resultados. 



\section{REFERENCIAS BIBLIOGRÁFICAS}

Aguirre Minvielle, Claudia. «Definición de prácticas de Apropiación Social de la Ciencia y de la Tecnología, historia de una conversación». Revista Ciencia, tecnología y democracia: Reflexiones en torno a la apropiación social del conocimiento, Memorias del Foro-Taller de Apropiación Social de la Ciencia, la Tecnología y la Innovación. Editado por Tania Pérez Bustos y Marcela Lozano Borda,177-184. Colombia: Universidad EAFIT. Colciencias, 2011.

Andrade, Jesús Alberto y Campo-Redondo, María. «Apropiación social de las tecnologías de información: políticas públicas para la participación ciudadana», Quórum Académico, Volumen 9, No 1 enero-julio 2012.Venezuela: Universidad del Zulia, 2012.

Dussel, Inés. "Aprender y enseñar en la cultura digital». VII Foro Latinoamericano de Educación; educación y nuevas tecnologías: los desafíos pedagógicos ante el mundo digital. Editado por Inés Dussel y Luis Alberto Quevedo. Buenos Aires: Santillana, 2011.

Diéguez, Alejandro. «El determinismo tecnológico: indicaciones para su interpretación». Argumentos de Razón Técnica N ${ }^{\circ}$ 8. España: Universidad de Málaga. 2005, disponible en: https://sociotecno7.files.wordpress. com/2009/09/determinismo-tecnologico.pdf

Freixes, Nuria G. y Parcerisa-Aran, Artur. «La intervención socioeducativa desde una mirada didáctica», Revista Edetania No 45 (2014): 5572, disponible en: https://dialnet.unirioja.es/servlet/articulo?codigo $=5010267$

De Querol, Ricardo (2016). «Zygmunt Bauman: «Las redes sociales son una trampa». El País, enero09,2016, disponible en: https://elpais.com/cultura/2015/12/30/babelia/1451504427_675885.html

Ministerio de Educación. «Plan de Acción del Programa Cerrando la Brecha del Conocimiento». El Salvador: MINED, 2009.

Organización de las Naciones Unidas para la Educación, la Ciencia y la Cultura (UNESCO). Las Tecnologías de la información y la comunicación en la enseñanza. Manual para docentes o cómo crear nuevos entornos de aprendizaje abierto por medio de las TIC. Francia: División de Educación Superior UNESCO, 2005. 
Organización de las Naciones Unidas para la Educación, la Ciencia y la Cultura (UNESCO). «La integración de las Tecnologías de la Información y Comunicación en los Sistemas Educativos». Argentina: Instituto Internacional de Planeamiento de la Educación IIPE-UNESCO, 2005.

Ortega, José. «Pedagogía Social, realidades actuales y perspectivas de futuro». Revista de Educación No 336 (2005): 111-127, disponible en: http:// www.revistaeducacion.mec.es/re336/re336_07.pdf

Vaillant, Denise. «Integración de TIC en los Sistemas de formación docente inicial y continua para la educación básica en América Latina». Argentina: Fondo de las Naciones Unidas para la Primera Infancia (UNICEF), 2013 . 